\section{Assesment of fundus autofluorescence in serpiginous and serpiginous-like choroidopathy}

\begin{abstract}
Aims The aim of this study was to correlate the activity status disclosed in fluorescein angiography (FA) and fundus autofluorescence (FAF) imaging, and the variations of FAF images in the evolution of serpiginous choroidopathy (SC) and serpiginous-like choroidopathy (SLC).

Methods Prospective consecutive case series. Patients with SC or SLC were included from July 2009 to December 2010. All patients underwent FAF imaging (Spaide Autofluorescence Filters, Topcon TRC 50IX) and FA (Topcon TRC 50IX).

Results Twelve patients (eight males, mean age 51.2 years) were included. Bilateral involvement in nine cases. Three different patterns of FAF images were present: active inflammation, transitional, and inactive inflammation.

Conclusion FAF may be a useful tool for following patients with SC and SLC. It is possible to reserve other invasive techniques, such as FA, for cases with suspicious activity disclosed by FAF imaging.

Eye (2012) 26, 1232-1236; doi:10.1038/eye.2012.121; published online 29 June 2012
\end{abstract}

Ophthalmology, Hospital Clínico Universitario de Valladolid, Valladolid, Spain

${ }^{2}$ IOBA (Instituto Universitario de Oftalmobiología), University of Valladolid, Valladolid, Spain

Correspondence: E Carreño, Department of Ophthalmology, Hospital Clínico Universitario de Valladolid, Avenida Ramón y Cajal s/n, E-47005 Valladolid, Spain

Tel: +34 983 420000; Fax: +34 983257511

E-mail: estherc@ioba.med. uva.es

Received: 15 November 2011

Accepted in revised form: 17 May 2012

Published online: 29 June 2012

This paper was presented at EVER 2010, Crete, Greece, where it won the poster prize in the Immunology/ Microbiology section.
Keywords: serpiginous choroidopathy; serpiginous-like choroidopathy; choroiditis; posterior uveitis; autofluorescence

\section{Introduction}

Serpiginous choroidopathy (SC) is a rare, bilateral, chronic, progressive, recurrent inflammation of the retinal pigment epithelium (RPE), choriocapillaris, and choroid. ${ }^{1-3}$ The aetiology is unknown, and it constitutes $<5 \%$ of posterior uveitis cases. ${ }^{4}$ Although it is usually defined as a bilateral disease, the typical presentation is a unilateral worsening of central vision, metamorphopsia, and/or scotoma. ${ }^{5}$
E Carreño ${ }^{1,2}$, A Portero², JM Herreras ${ }^{1,2}$

and MI López ${ }^{1}$
The disease begins with ill-defined patches of creamy yellow sub-retinal infiltrates in the peripapillary area. It progresses in an irregular serpentine fashion centrifugally. Active lesions resolve leaving an area of atrophy involving both the choriocapillaris and the RPE. The disease is characterised by multiple recurrences at variable intervals. Each new episode of activity leaves a bigger serpentine extension of atrophied choriocapillaris and RPE. As a result of the multiple recurrences and in spite of treatment, up to $25 \%$ of the eyes have a final visual acuity less than $20 / 200{ }^{6}$ Although a definitive treatment modality is not available for this disease, steroids and immunomodulatory therapies seem to be effective in inducing remission of inflammatory activity.

Similarly, cases associated with systemic disease, both infectious and autoimmune, ${ }^{7-12}$ have been published with modifications of the diagnostic criteria for the classic concept of SC. These atypical cases that resemble SC are denominated under the general term of serpiginous-like choroidopathy (SLC).

Fluorescein angiography (FA) is one the most useful techniques currently used to identify active inflammatory disease. This technique, however, is not exempt of risks, including serious allergic responses. ${ }^{13}$ In contrast, fundus autofluorescence (FAF) imagery is a useful non-invasive tool for detecting RPE changes in acute episodes of SC or SLC. ${ }^{14}$ FAF permits topographic mapping of lipofuscin distribution in the RPE cell monolayer. Excessive accumulation of lipofuscin represents a common pathogenic pathway in various complex retinal diseases and is believed to precede photoreceptor degeneration. ${ }^{15-17}$

The aim of this pilot study was to correlate FAF images with the inflammatory activity of the disease. This will establish the basis for the 
use of FAF imagery as a convenient tool in the follow-up of patients with SC. Importantly, it avoids the use of other invasive techniques, such as FA, which can then be reserved for cases in which suspicious activity is disclosed by FAF imaging. Determining the activity status of the inflammatory disease is crucial to offering correct and prompt treatment in an effort to avoid severe visual impairment. FAF imagery provides quick, economical, and useful information for follow-up without any associated risk.

\section{Patients and methods}

Patients of the Ocular Immunology Unit of the 'Hospital Clínico Universitario de Valladolid' with SC or SLC were prospectively and consecutively included from July 2009 to December 2010. This study followed the tenets of the Declaration of Helsinki. The protocol was approved by the Institutional Review Board, and all patients signed an informed consent to be included.

All patients received a comprehensive ophthalmic examination, including visual acuity, slit-lamp biomicroscopy, and dilated funduscopic examination. Fundus photography and FAF imaging were performed in different stages of the disease to determine the progression or the stability of the lesions. FA was also performed to correlate the inflammatory status of the disease with the FAF imagery.

For FAF imaging, the Topcon TRC 50IX retinal camera with Spaide Autofluorescence Filters (Topcon, Paramus, NJ, USA) was used with an excitation wavelength of $585 \mathrm{~nm}$ (bandwidth 550-600 $\mathrm{nm}$ ) and an emission wavelength of $695 \mathrm{~nm}$ (bandwidth $660-800 \mathrm{~nm}$ ). Images were obtained using OIS WinStation 5000 software (Ophthalmic Imaging System, Sacramento, CA, USA) and analysed using Adobe Photoshop 5.5 (Adobe Systems Incorporated, San Jose, CA, USA). For FA imaging the Topcon TRC 50IX retinal camera was used.

The diagnosis of SC was made based on the clinical appearance and FA findings. Systemic studies were performed to rule out other conditions that could mimic SC, such as tuberculosis, sarcoidosis, or lupus. Those patients in which systemic disease was present were diagnosed with SLC. They were also included in the study according to the presence of similar FAF and FA patterns.

For FA, a lesion was established as active when there was a blockage of fluorescein early and a progressive diffuse staining and leakage in the late frames. In the atrophic areas, the main findings were early hypofluorescence secondary to atrophy of choriocapillaris and progressive hyperfluorescence at

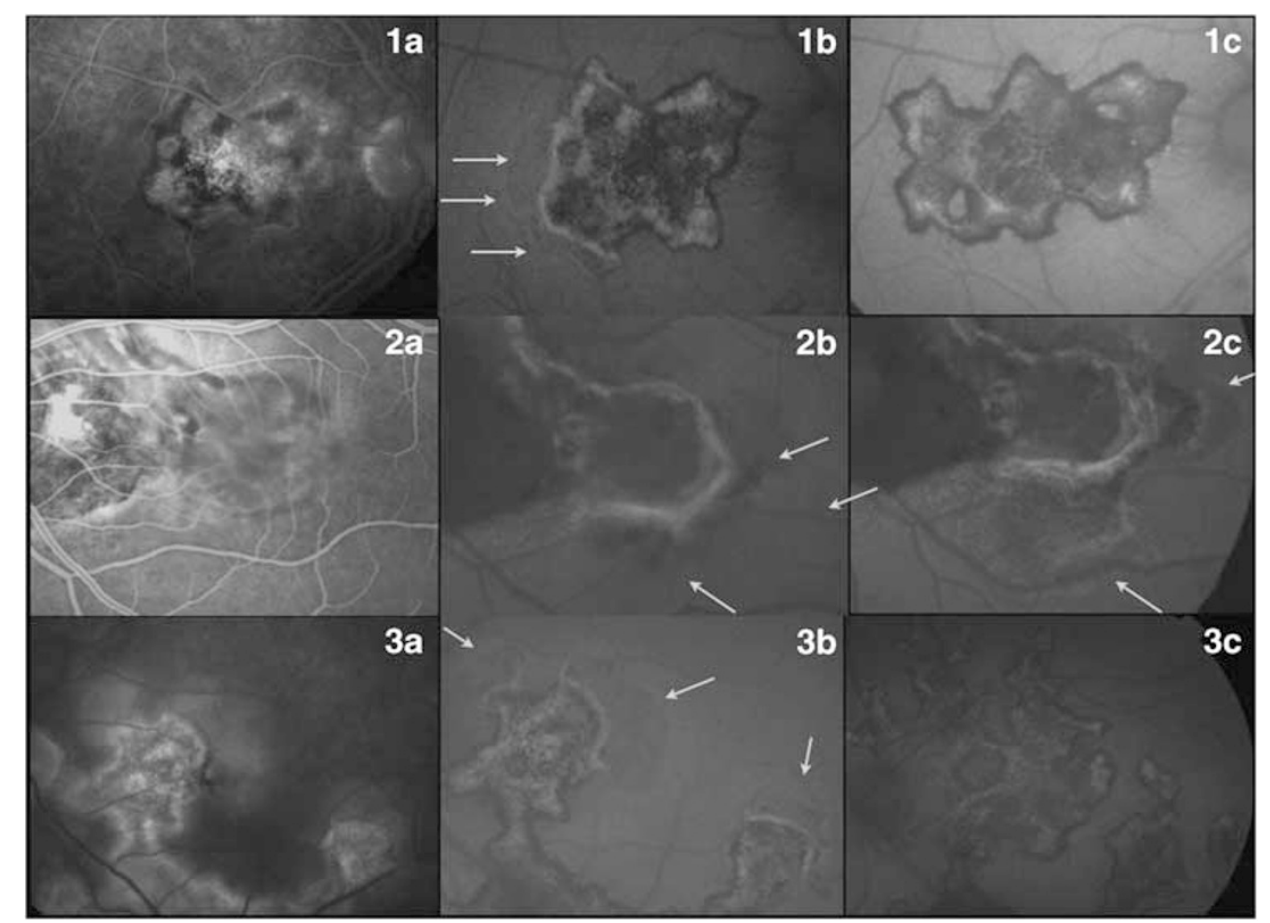

Figure 1 FA and FAF images in three cases of SC with active inflammation. Three patients (rows 1-3) had FA activity (a). FAF images were taken at baseline (b) and 1 month later (c). The FAF was characterised by dark areas surrounding hyperautofluorescent edges. The border of the active lesions is labelled with arrows. These images correlated well with hypofluorescent areas in FA indicating blockage of the fluorescein and late staining of the active areas. Changes between baseline images (b) and those at 1 month (c) showed the progression of RPE destruction. 
the margins of the lesion with eventual diffuse late staining of the underlying sclera and surrounding fibrosis.

\section{Results}

Nine patients with SC and three with SLC (21 eyes) were included in the study. The mean age was 51.2 years (range: 36-77 years) and included eight males and four females. Both eyes were affected in nine patients, and a single eye in three patients with SLC. FA and FAF imaging were performed in each case. The mean follow-up was 8.8 months (range: 6-14 months).

When the angiogram disclosed activity (three cases), the FAF imagery showed a characteristic hypoautofluorescence surrounding the hyperautofluorescent edges of the lesions with a clear progression of the area of atrophy in the follow-up at 1 month, inactivation in the FA and transitional pattern in the FAF (discussed later) (Figure 1). This pattern of FAF was seen only in patients with activity signs in FA.

When FA did not disclose inflammatory activity two different FAF patterns were apparent. First, the FAF pattern was transitional with hypoautofluorescence at the surrounding borders of the central hyperfluorescent areas (Figure 2). In these eyes, the area of atrophy was stable in the FAF at the 6-month follow-up with lack of the autofluorescence. Second, there was a pattern with hypoautofluorescent lesions both at the centre and at the edges of the lesions (Figure 3), and this pattern remained stable in the 6-month follow-up.

\section{Discussion}

SC presents a wide spectrum in its clinical appearance. ${ }^{5}$ Similarly, the spectrum of autofluorescence images is also diverse, although different autofluorescence patterns are associated with the current activity and the evolution of the disease. ${ }^{14}$ Our findings are congruent with those described by Cardillo Piccolino et al, ${ }^{14}$ with stages closely associated inflammatory inactivation. First there is FAF diffuse hypoautofluorescence that surrounds the lesions. This hypoautofluorescence is probably consequence of oedema of the deep retina or RPE as has been previously proposed. ${ }^{14}$ This pattern of FAF is associated with the presence of active inflammation as indicated by FA.

Afterwards a sharp hypoautofluorescence border surrounds the hyperautofluorescent lesions. We believe that this represents a transition to inactive inflammatory disease. Finally, the lesions become totally hypoautofluorescence (inactive) in the final FAF images.

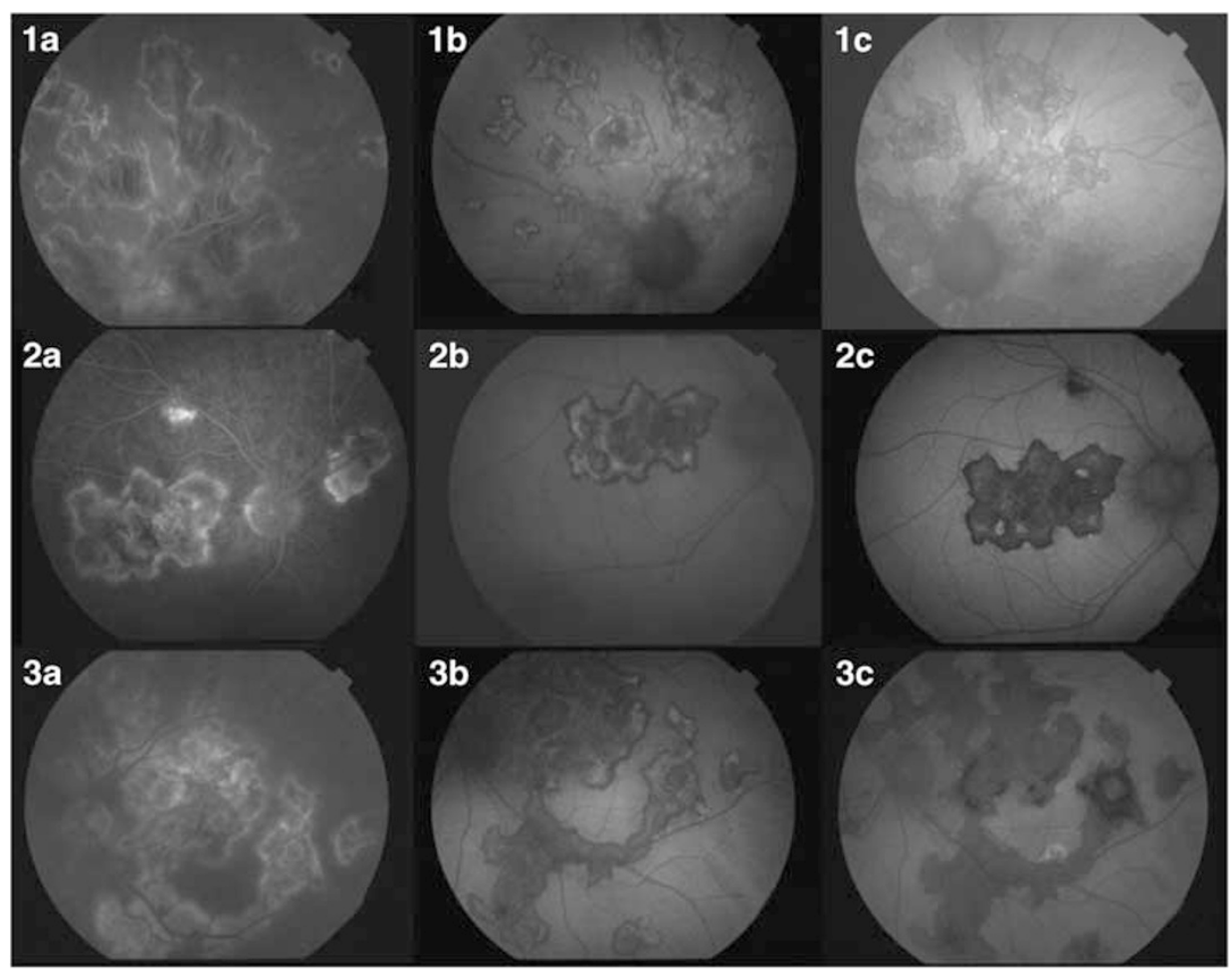

Figure 2 FA and FAF images in three cases of SC in a transitional state. These three patients (rows 1-3) did not have FA activity (a). The baseline FAF showed a transitional status characterised by hypoautofluorescence at the edges of central hyperautofluorescent areas (b). 1 month later (c) the FAF image showed that the lesion had become inactive. 


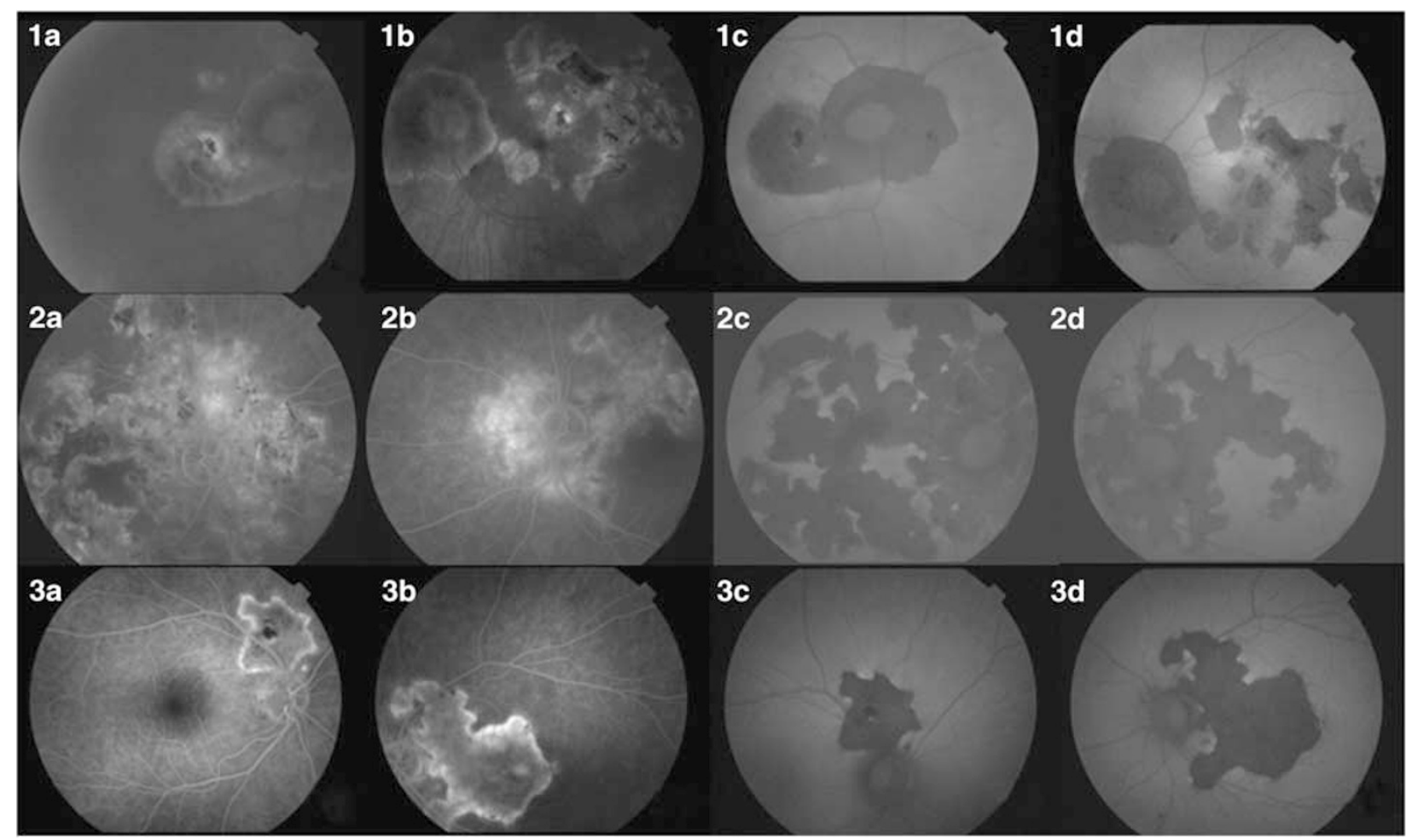

Figure 3 FA and FAF images in three cases of bilateral inactive SC. These three representative patients (rows 1-3) did not have FA activity ( $a$, right eye; b, left eye). FAF imagery (c, right eye; $d$, left eye) showed hypoautofluorescence in the affected areas.

Further studies are warranted, but based on the current study, we suggest the following autofluorescence patterns in SC: (1) active inflammation is characterised by a hypoautofluorescent halo that surrounds the edges of hyperautofluorescent lesions. This pattern correlates with active inflammation as assessed by FA. The lesion is fast growing, and with time can extend beyond the hypoautofluorescent halo. (2) Transitional SC is an intermediate stage between active and inactive inflammation. FA indicates that most or all of the inflammation has subsided. FAF images show a hypoautofluorescent line that surrounds all edges of the hyperautofluorescent lesions. The FAF images indicate that the SC lesions are stable and do not tend to grow. (3) Inactive inflammation is characterised by FAF images of lesions that are dark, revealing complete loss of fluorophores, with very sharp borders. There is no hyperautofluorescence at the edges. This pattern correlates with inactive inflammation in FA and lesions that are stable over time, while the inflamatory activity remains inactive.

The most common and visually significant ocular complication associated with SC is choroidal neovascularization. ${ }^{18}$ It occurs in $13-35 \%$ of the patients ${ }^{19}$ and usually leads to a worsening of vision. In this case, FAF is not sufficient to monitor the activity of the disease, and FA is necessary.
In conclusion, FAF is an easy, non-invasive, and useful tool to evaluate the extent of the area affected by SC and serpiginous-like diseases. Subsequently, it also could be useful in following the evolution of the disease, to determine the risk of progression of the lesions, and to evaluate the inflammatory activity, while avoiding invasive procedures. However, FA will continue to be essential in cases where choroidal neovascularization is suspected.

\section{Summary}

\section{What was known before}

- Serpiginous and serpiginous-like choroidopathy are a very severe bilateral choroiditis, which usually require an aggressive treatment. The gold-standard to disclose the activity status currently is fluorescein angiography. Fundus autofluorescence is cheaper and safer than fluorescein angiography and it has been used to monitor other pathologies such as dry age-related macular degeneration.

\section{What this study adds}

- Fundus autofluorescence can be an appropriate tool to monitor patients with serpiginous and serpiginous-like choroidopathy. It can disclose the activity status, reserving fluorescein angiography for cases where choroidal neovascularization is suspected. 


\section{Conflict of interest}

The authors declare no conflict of interest.

\section{References}

1 Bacin F, Larmande J, Boulmier A, Juillard G. [Serpiginous choroiditis and placoid epitheliopathy]. Bull Soc Ophtalmol Fr 1983; 83(10): 1153-1162.

2 Abu el-Asrar AM. Serpiginous (geographical) choroiditis. Int Ophthalmol Clin 1995; 35(2): 87-91.

3 Ciulla TA, Gragoudas ES. Serpiginous choroiditis. Int Ophthalmol Clin 1996; 36(1): 135-143.

4 Chang JH, Wakefield D. Uveitis: a global perspective. Ocul Immunol Inflamm 2002; 10(4): 263-279.

5 Lim WK, Buggage RR, Nussenblatt RB. Serpiginous choroiditis. Surv Ophthalmol 2005; 50(3): 231-244.

6 Christmas NJ, Oh KT, Oh DM, Folk JC. Long-term followup of patients with serpinginous choroiditis. Retina 2002; 22(5): 550-556.

7 Vonmoos F, Messerli J, Moser HR, Prunte C, Flammer J, Haefliger IO. Immunosuppressive therapy in serpiginous choroiditis-case report and brief review of the literature. Klin Monbl Augenheilkd 2001; 218(5): 394-397.

8 Gupta V, Gupta A, Arora S, Bambery P, Dogra MR, Agarwal A. Presumed tubercular serpiginouslike choroiditis: clinical presentations and management. Ophthalmology 2003; 110(9): 1744-1749.

9 Fuentes-Paez G, Celis-Sanchez J, Torres J, Martinez-Osorio H, Herreras JM. Serpiginous choroiditis in a patient with systemic lupus erythematosus. Lupus 2005; 14(11): 928-929.
10 Edelsten C, Stanford MR, Graham EM. Serpiginous choroiditis: an unusual presentation of ocular sarcoidosis. Br J Ophthalmol 1994; 78(1): 70-71.

11 Mahendradas P, Kamath G, Mahalakshmi B, Shetty KB. Serpiginous choroiditis-like picture due to ocular toxoplasmosis. Ocul Immunol Inflamm 2007; 15(2): 127-130.

12 Sonika Narang S, Kochhar S, Srivastava M, Gupta R, Sood S. Posterior scleritis mimicking macular serpiginous choroiditis. Indian J Ophthalmol 2003; 51(4): 351-353.

13 Lu VH, Ho IV, Lee V, Hunyor AP. Complications from fluorescein angiography: a prospective study. Clin Experiment Ophthalmol 2009; 37(8): 826-827.

14 Cardillo Piccolino F, Grosso A, Savini E. Fundus autofluorescence in serpiginous choroiditis. Graefes Arch Clin Exp Ophthalmol 2009; 247(2): 179-185.

15 Wing GL, Blanchard GC, Weiter JJ. The topography and age relationship of lipofuscin concentration in the retinal pigment epithelium. Invest Ophthalmol Vis Sci 1978; 17(7): 601-607.

16 Weiter JJ, Delori FC, Wing GL, Fitch KA. Retinal pigment epithelial lipofuscin and melanin and choroidal melanin in human eyes. Invest Ophthalmol Vis Sci 1986; 27(2): 145-152.

17 Dorey CK, Wu G, Ebenstein D, Garsd A, Weiter JJ. Cell loss in the aging retina. Relationship to lipofuscin accumulation and macular degeneration. Invest Ophthalmol Vis Sci 1989; 30(8): 1691-1699.

18 Prost M. Results of long-term observations of patients with serpiginous choroidopathy. Klin Oczna 1993; 95(8): 306-309.

19 Blumenkranz MS, Gass JD, Clarkson JG. Atypical serpiginous choroiditis. Arch Ophthalmol 1982; 100(11): 1773-1775. 